\title{
RESEARCH ON ELECTRICAL TESTING AND CHEMICAL ANALYSIS OF ELECTRIC VEHICLE LITHIUM BATTERY PACKS
}

\author{
Li Cai*, Peng-qiu Hu, Ze-fu Tan \\ Chongqing Three Gorges University, Signal and Information Processing Key Lab, Wanzhou 404000, \\ Chongqing City, China. \\ *Corresponding author: Li Cai \\ Email: licailicai28193@126.com
}

\begin{abstract}
The lithium battery is an important part of the electric vehicle. In order to analyze the performance of the electric vehicle, the lithium battery must be tested. The paper introduces the chemical reaction process and working principle of the lithium battery packs, and analyses the requirement and principle of the lithium battery test at first. Second, an new algorithm for the internal resistance test of model parameter identification has been put forwarded, the process and parameters of the new algorithm has been detailed clearly. Then, the paper gives the working principle of the hardware circuit of the test equipment and the workflow of the software. The core of the test equipment is to control AC current in lithium battery by STM8S003 embedded microcontroller, and to measure the real time current, voltage, discharge curve and internal resistance of lithium battery through parameter identification algorithm. At last, the lithium battery packs which made of 818650 type lithium battery was taken as an example, and the test results were analyzed.
\end{abstract}

Keywords: Electrical Testing, Chemical Analysis, Battery Packs.

\section{Introduction}

Lithium battery is a kind of battery which is made of lithium metal or lithium alloy as negative electrode and uses non water electrolyte solution. In 1912, lithium metal battery were first proposed and studied by Gilbert N. Lewis. In 1970s, M. S. Whit Ingham proposed and began to study lithium ion battery. Because the chemical properties of lithium metal are very active, the processing, preservation and use of lithium metal are very demanding for the environment.

Lithium battery can be roughly divided into two types: lithium metal battery and lithium ion battery. Lithium ion battery does not contain a metal state of lithium, and can be rechargeable. The fifth generation of rechargeable battery, the lithium metal battery, was born in 1996, and its safety, specific capacity, self discharge rate and performance price ratio are all better than that of lithium battery.

Lithium-ion battery has the characteristics of light weight, large energy storage, high power and no pollution. It has been applied more and more widely in various fields, and has made great progress in research and production. Lithium ion battery has become a new trend in electric vehicle development as a power source in electric vehicle.

The advancement of lithium ion battery technology and the application in EV have stimulated the worldwide research and development boom, so the lithium ion battery will play an important role in the field of electric vehicles and new energy. At present, in the electric vehicle, the lithium ion battery is widely used as the lithium iron phosphate battery, its thermal stability and safety are better, at the same time the price is relatively cheap. These factors make lithium phosphate lithium-ion battery the first choice for small electric vehicles and PHEV power cells. However, in lithium ion battery, the specific energy, specific power and operation voltage of lithium phosphate battery are relatively low. In the application of large pure electric vehicle, lithium cobalt oxide and lithium magnate battery have more advantages. For example, the power battery of Tesla Model S85 electric vehicle consists of 700018650 type lithium batteries [1].

During the use of lithium battery packs, users often judge and detect the performance state of lithium battery packs by parameters such as OCV, available capacity, internal resistance, load discharge curve, self discharge curve and so on. Among them, internal resistance and discharge curve are the most important reference indexes for lithium battery packs. The internal resistance can directly react with the SOH of the lithium battery packs, and the discharge curve can directly reflect the performance of the lithium battery packs. The internal resistance of the lithium battery packs is not an ordinary ohm resistance. It is made up of the ohm internal resistance and the polarization internal resistance. It 
can not be measured directly by the multi-meter. How to accurately, quickly and conveniently measure the internal resistance and discharge curve of lithium battery is a hot spot in the field of industrial application and scientific research in the battery field.

The research on internal resistance of lithium battery packs at home and abroad is generally used to estimate the internal resistance of lithium battery packs. A method for estimating internal resistance of lithium battery packs: HPPC is proposed in reference [2]. This method detects the parameters of lithium battery packs during pulse loading and transition, and then brings it into the PNGV model to estimate the internal resistance, and achieves ideal results in the case of model adaptation. Reference put forwarded a new Thevenin model for lithium battery packs internal resistance estimation is proposed. The internal resistance and internal resistance of the lithium battery packs are changed from constant to variable, which is better than the traditional Thevenin model. However, in the existing literature, the measurement of internal resistance of lithium battery packs is mostly based on Simulation and algorithm, and there are few literature's on the whole solution of the test instrument, and the existing algorithm is difficult to implement and the cost is high.

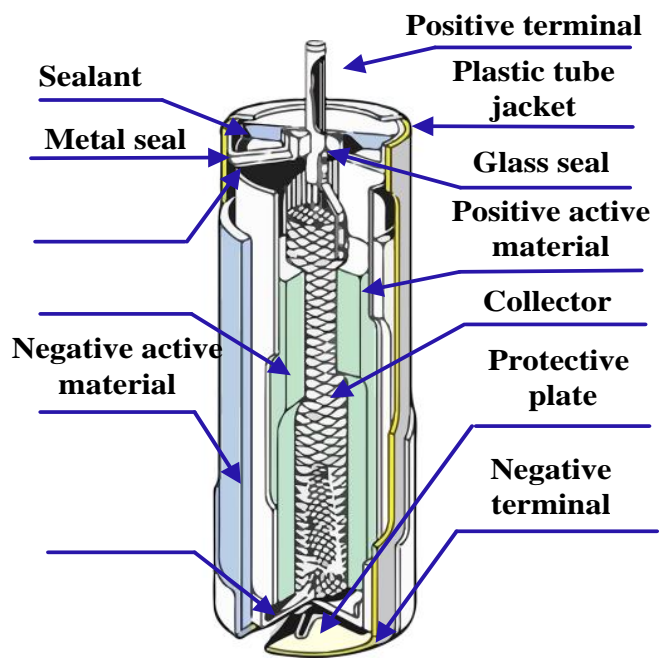

(a) Lithium battery structure

\section{Working Principle of Lithium Battery Packs \\ 2.1 Chemical reaction}

The cathode material of the lithium battery packs is lithium cobalt oxide, and the negative electrode is carbon.

The principle of lithium battery packs is the principle of charge discharge. When the lithium battery packs is charged, the lithium ion is generated on the positive pole of the battery packs, and the lithium ion generated by the electrolyte moves to the anode through the electrolyte. As a negative electrode, carbon has a layered structure. It has many pores. The lithium ion to the negative electrode is embedded in the pores of the carbon layer. The more lithium ions are embedded, the higher the charging capacity. In the same way, when the battery packs is discharged, the lithium ion embedded in the negative carbon layer moves out and moves back to the positive pole. The more lithium ion in the positive pole, discharge capacity is higher. The battery packs capacity we usually say refers to the capacity of the discharge.

In a word, during the charge and discharge process of lithium battery packs, the lithium battery packs is in the state of movement from positive to negative to positive pole.

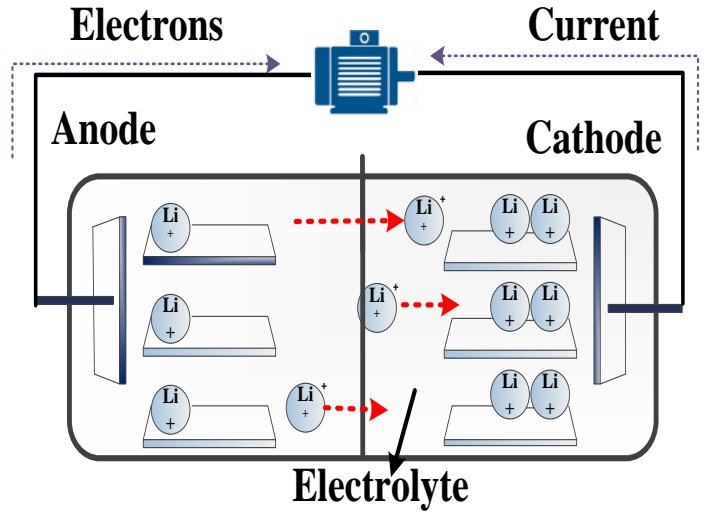

(b) Chemical reaction

Figure.1 Structure and Chemical reaction lithium battery packs

The charging and discharging process of the lithium battery packs is a reversible chemical reaction process, the equation is as follows, formula (1) the chemical reaction of the positive pole, formula (2) the chemical reaction of the negative electrode, and formula (3) the complete chemical reaction.

$$
\begin{aligned}
& 6 \mathrm{C}+\mathrm{Li}+e^{-}=6 \mathrm{CLi} \\
& \mathrm{LiCoO}_{2}=\mathrm{CoO}_{2}+\mathrm{Li}+e^{-} \\
& 6 \mathrm{C}+\mathrm{LiCoO}_{2}=\mathrm{CoO}_{2}+6 \mathrm{CLi}
\end{aligned}
$$

Lithium battery packs consists of three parts: the positive electrode, negative electrode, diaphragm. During the discharging process, the lithium ion of the negative electrode diffuses to the surface of the active material and undergoes a chemical reaction. During the charging process, the lithium ions reach the positive electrode through the diffusion of the electrolyte and the conductance of ions.

Finally, the charged ions diffuse into the inland region of the metal oxide active. The long and short separators are an electrical insulator that allows 
electrons to follow the opposite direction through an external circuit or load. The two composite electrodes contain sticky caking agents and fillers to improve the transmission of electrons throughout the solid substrate [3]. Charge and discharge is completed, accompanied by the voltage on rise or decay, in the curing phase, the concentration of lithium ions concentrated on both ends of the depletion or saturation, in the electrolysis stage, Lithium ions are depleted near the poles.

\subsection{Lithium battery packs}

The charge and discharge of a single lithium battery, a reversible chemical reaction, a side reaction and a main reaction, will cause the loss of the lithium battery as Figure 2.

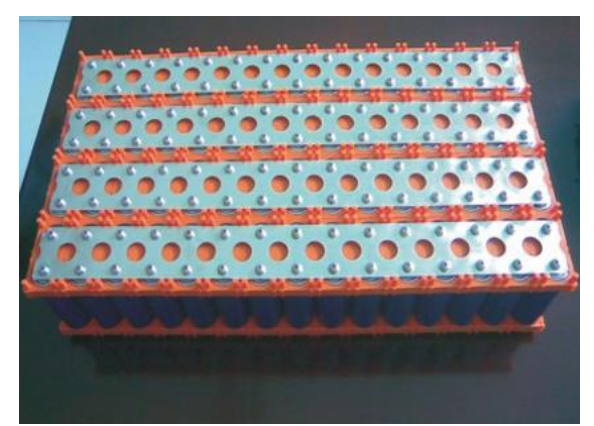

(a) lithium battery packs of EV
Practical electric car lithium battery, composed of many single cells, repeated charge and discharge after the wear extent of each single cell is different, resulting in differences in the lithium battery packs of single cell, accelerate the decay of the battery capacity, resulting in electric car battery life is short.

On the other hand, due to different environment, processing differences, single battery materials production process and the use of different number of single battery comprising a battery, but also one of the leading reasons, lithium battery has the difference of the number, possibly caused by the difference of monomer is higher. The most direct effect of the difference in lithium battery is to shorten the life of the electric vehicle lithium battery packs.

Figure. 2 Lithium battery packs

\section{Test of Lithium Battery Packs 3.1 Test parameters}

The lithium battery packs has six main parameters, such as Battery capacity, nominal voltage, charging termination voltage, discharge termination voltage, internal resistance and self discharge ratio.

Battery capacity: the capacity of the battery is determined by the number of active substances in the battery, usually expressed in unit as mAh or Ah .

Nominal voltage: potential difference between positive and negative electrode of the battery according to nominal voltage of battery. The nominal voltage is determined by the electrode potential of the plate material and the concentration of the internal electrolyte. The discharge curve of a lithium battery packs is a parabola. Taking 18650 lithium batteries for example, $4.3 \mathrm{~V}$ drops to $3.7 \mathrm{~V}$ and $3.7 \mathrm{~V}$ down to $3.0 \mathrm{~V}$, all of which are very fast. Only about $3.7 \mathrm{~V}$ has the longest discharge time, almost occupying the time of $3 / 4$, so the nominal voltage of lithium battery is the voltage that maintains the longest discharge time.

Charging termination voltage: after the lithium battery pack is completed, the active material on the pole plate has reached saturation state, and then the battery will continue to charge, and the voltage of

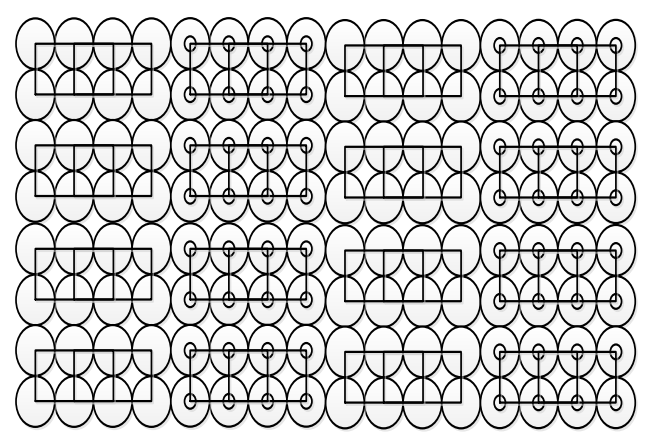

(b) lithium battery packs Parallel connection the battery will not rise. The voltage is called the termination voltage of the battery.

Discharge termination voltage: the discharge termination voltage refers to the minimum voltage allowed when the battery is discharged. The discharge termination voltage is related to the discharge rate. Generally speaking, the discharge termination voltage of the 18650 lithium battery is $2.7 \mathrm{~V}$.

Internal resistance: the internal resistance of the battery is determined by the resistance of the plate and the impedance of the ion flow. During the charging and discharging process, the resistance of the image engine and the plate is constant, but the impedance of the ion flow will change with the increase of electrolyte concentration and charged ion. The internal resistance will increase with the decreases of lithium battery packs OCV voltage. Therefore, when charging at low voltage, we need to pre charge first, so as to prevent the current from being too large and cause the excessive calorific value of the battery.

Self discharge ratio: it refers to the percentage of the battery that is automatically lost in a period of time when the battery is not used. In general, the self discharge rate of lithium ion battery is $5 \%-8 \%$ at normal temperature. 


\subsection{AC injection method}

According to the international standard "IEC61960:2003", AC signal generated by AC power $I(t)=I_{\max } \sin (2 \pi f t), I(t)$ is injected into two poles of lithium battery, the internal resistance of lithium battery is generated by $I(t)$, resulting in voltage $U(t)=U_{\max } \sin (2 \pi f t+\Phi)$, obviously, the frequency is the same and the phase is different [4].

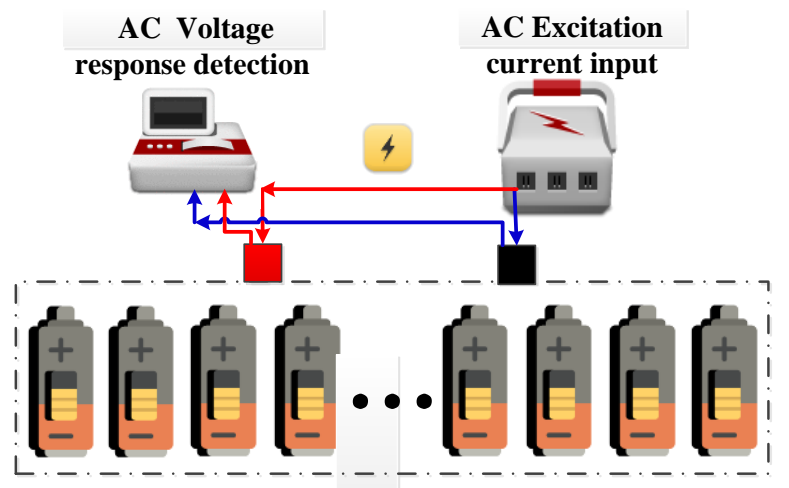

Lithium battery packs

Figure. 3 AC injection method

\subsection{Parameter identification}

System identification is an advanced modeling tool ideal for engineering design and data analysis within the Software, Intelligent Systems and Modeling Program [5].

When lithium batteries work online, the initial state $\left({ }^{t=t_{0}}\right)$ electrochemical reaction has not yet occurred, and the internal resistance is composed of ohmic internal resistance. The internal resistance of the later $\left(t>t_{0}\right)$ is composed of three kinds of internal resistance, polarization internal resistance and concentration polarization internal resistance, and the polarization internal resistance changes exponentially with [6]. The formulas for obtaining internal resistance according to figure 2 and Ohm's law are as follows:

$$
\begin{aligned}
& R_{0}=\frac{U\left(T_{0}\right)-U(t)}{I(t)} t=T_{0} \\
& R_{0}=\frac{U\left(T_{0}\right)-U(t)-I(t) \times R_{1} e^{\frac{t-T_{0}}{\tau}}}{I(t)} \\
& t>T_{0}
\end{aligned}
$$

The time constant is the identification parameters; it reflects the length of the polarized capacitor charging process.
After $\mathrm{t}=\mathrm{t} 0$ time, set any time point ${ }^{t_{1}}$ and ${ }^{t_{2}}$, the battery output port voltage is $U\left(t_{1}\right)$ and $U\left(t_{2}\right)$, there is the next type (2).

$$
\tau=\frac{\left(t_{2}-t_{1}\right) \times I(t)}{\ln \left[U_{0}(T)-I(t) \times R_{0}-U\left(t_{1}\right)\right]-\ln \left[U_{0}(T)-I(t) \times R_{0}-U\left(t_{2}\right)\right]}
$$

The parameter identification problem is the inability in principle to identify a best estimate of the value(s) of one or more parameters in a regression. This problem can occur in the estimation of multipleequation models where the equations have variables in common. More generally, the term can be used to refer to any situation where a statistical model will invariably have more than one set of parameters that generate the same distribution of observations, meaning that multiple parameterizations are observation-ally equivalent [7]. Considering the speed of the lithium battery's polarization reaction and the accuracy of the measurement, the intervals of $t_{1}$ and $t_{2}$ are set to within $5 \mathrm{~ms}$ [8].

\section{Test Experiment and Result Analysis 4.1 Test experimental platform}

The main controller selects STM8S003 embedded micro controller, single-chip microcomputer through PWM (pulse width modulation) calculation, built-in 10 bit D/A (digital / analog conversion) output $1(t)$. T(t) is output from the PD4 pin, through the SS8550 Trident and the switch diode BAW56W, finally acts on LY8205S dual channel N field-effect transistor, so as to control the size of lithium battery test current $I(t)$. Considering that the discharge current is large enough to generate high heat, it may reach more than $212{ }^{\circ}$. Conventional resistors usually cannot work at this temperature. So $7 \mathrm{~W} 2.2 \Omega \mathrm{J}$ cement resistance has been chosen as the heat source.

The port voltage of lithium battery packs is input from PD3 port to STM8S003 embedded micro controller, and is acquired after the 10 bit A/D (Analog/Digital)built by MCU.

YLB3641BH four Yang digital tubes displays the current value of current and voltage control switch, key switch SW-PB display content.

The system is powered by Mini USB interface, and the USB port voltage of $+5 \mathrm{~V}$ is stabilized at $3.3 \mathrm{~V}$ to drive the system by the three terminal voltage regulator module of PL3500. PD5 and PD6 pins are used as serial communication TXD (Transmit Data), RXD (Receive Data) pins, and communication with the host computer. 


\subsection{Experimental process and results}

The test object is a group of electric vehicle lithium battery packs. A set of lithium batteries consists of a 2418650 lithium batteries which type is NK16L072 and capacity is $200 \mathrm{mAh}$. Realization the test platform According to section 4.1 such as Figure 4.

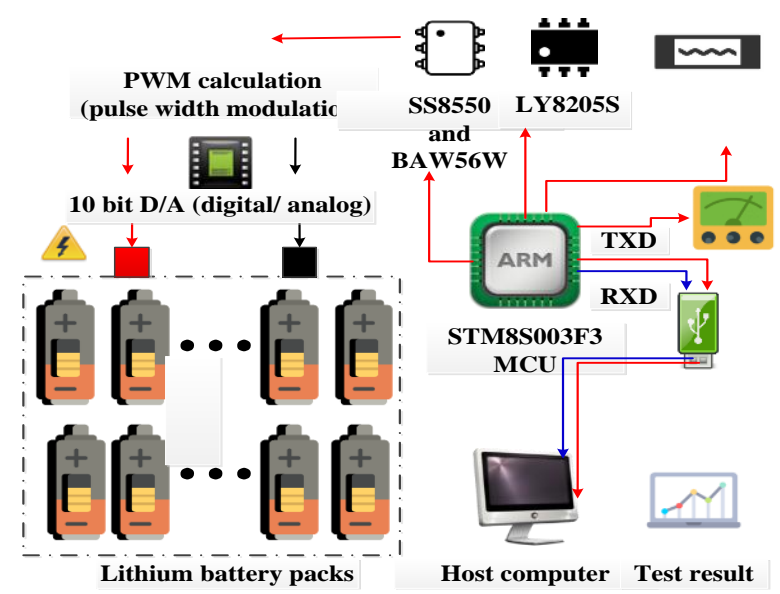

Figure. 4 Lithium battery packs test platform
The discharge current is set to $1000 \mathrm{~mA}$ and the discharge termination voltage is $76.8 \mathrm{~V}$. The AT526B type battery internal resistance tester is used as the contrast test equipment.

The parameters of the discharge curve and internal resistance and so on of the lithium battery packs were measured successfully as table I and Figure 5.

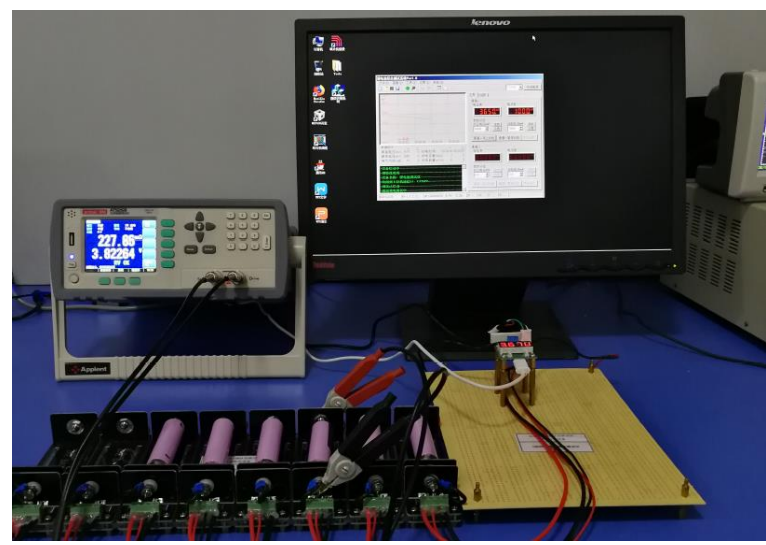

Figure. 5 Experimental test platform

Table 1 Test results

\begin{tabular}{|c|c|c|c|c|}
\hline Battery packs parameters & Unit & Test platform & AT526B & $\begin{array}{c}\text { Difference } \\
\text { ratio }\end{array}$ \\
\hline internal resistance & $\mathrm{m} \Omega$ & 766.72 & 758.8 & $1.03 \%$ \\
\hline output voltage & $\mathrm{V}$ & 102.184 & 105.830 & $3.57 \%$ \\
\hline capacity & $\mathrm{Ah}$ & 50.128 & 52.158 & $4.05 \%$ \\
\hline Discharge time & $\mathrm{Hour}$ & 18.21 & $/$ & $/$ \\
\hline Discharge energy & $\mathrm{Wh}$ & 191.98 & $/$ & $/$ \\
\hline
\end{tabular}

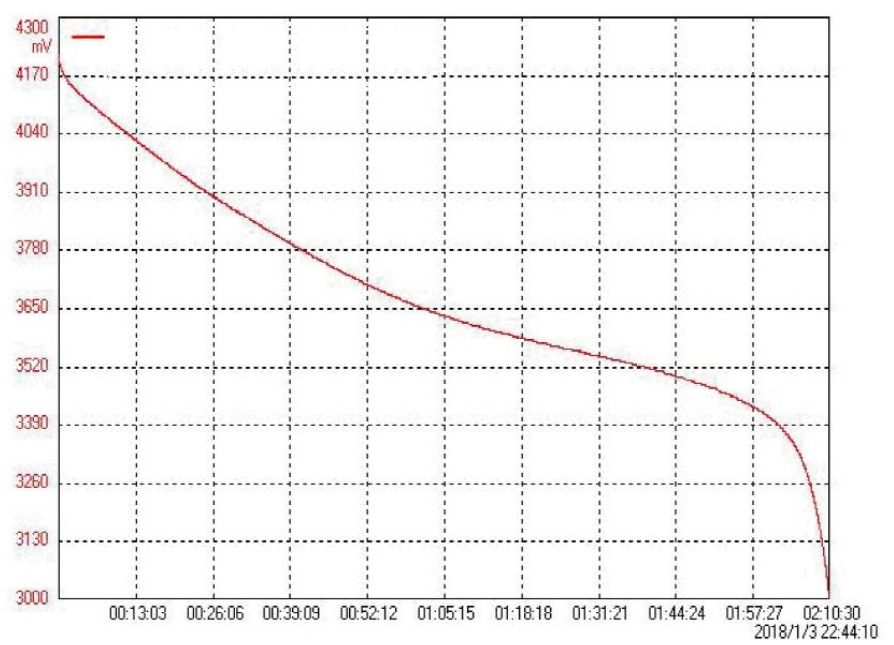

Figure. 6 Discharge curve of monomer 18650 lithium battery

\subsection{Result analysis}

Analyzing the data and table of the 4.2 chapters, following conclusions can are obtained:

For test methods and tests experimental platform, the scheme of this paper can accurately measure the internal resistance, output voltage, discharge capacity of lithium battery packs, can measure the discharge time, discharge energy and other professional testing equipment cannot measure the parameters of lithium battery packs, the measurement process is safe and reliable, discharge 
temperature rise can cause fever enough to control in a safe range, model parameter identification of the AC injection method practical effect is good, the test results of lithium battery packs tester based on the algorithm are accurate.

For the performance of lithium battery packs, the lithium battery packs can achieve high capacity utilization when the capacity and internal impedance are matched ideally. The more the number of parallel batteries in the short plate battery, the higher the end current of the short plate battery, with the increase of the number of parallel cells, the parameters of the battery pack checkbook begin to show normal distribution, and the extreme currents begin to decrease and tend to be stable [9].

The charging and discharging mode of shallow charging is advantageous to the optimization of lithium ion battery in parallel, and prolong the But it reduces the amount of utilization and increases the cost. The connection between the first string and the direct parallel battery can effectively reduce the imbalance of the current, and the number of batteries, the difference is smaller.

\section{Conclusion}

The development of lithium batteries and battery testing technology of mutual influence, mutual promotion, based on the actual needs from the battery development, a number of key areas of the lithium battery were studied, including battery detection, performance analysis, material synthesis improvement and application as a battery electrode.

The electrochemical performance parameter test system of lithium ion battery is introduced in this paper, which can realize the comprehensive test and analysis of the electrochemical performance of lithium battery.

Considering the versatility to battery research, the system can not only meet the detection and quality evaluation of the general performance of lithium batteries. The system only needs to change the corresponding test setup or a little expansion, and can also realize general measurement for other batteries and energy storage devices.

\section{References}

[1] Zhou, F., \& Lv, Z. (2017). A novel charging/discharging balancing strategy for lithium battery pack based on mixed charging mode. IEEJ Transactions on Electrical and Electronic Engineering, 12(4), 615-622.

[2] Zou, Y., Hu, X., Ma, H., \& Li, S. E. (2015). Combined state of charge and state of health estimation over lithium-ion battery cell cycle lifespan for electric vehicles. Journal of Power Sources, 273, 793-803.

[3] Ecker, M., Gerschler, J. B., Vogel, J., Käbitz, S., Hust, F., Dechent, P., \& Sauer, D. U. (2012). Development of a lifetime prediction model for lithium-ion batteries based on extended accelerated aging test data. Journal of Power Sources, 215, 248-257.

[4] Ouyang, M., Chu, Z., Lu, L., Li, J., Han, X., Feng, X., \& Liu, G. (2015). Low temperature aging mechanism identification and lithium deposition in a large format lithium iron phosphate battery for different charge profiles. Journal of Power Sources, 286, 309-320.

[5] Ding, F., Xu, W., Graff, G. L., Zhang, J., Sushko, M. L., Chen, X., ... \& Liu, X. (2013). Dendrite-free lithium deposition via self-healing electrostatic shield mechanism. Journal of the American Chemical Society, 135(11), 4450-4456.

[6] Weng, C., Sun, J., \& Peng, H. (2014). A unified open-circuit-voltage model of lithium-ion batteries for state-of-charge estimation and stateof-health monitoring. Journal of power Sources, 258, 228-237.

[7] He, Z., Gao, M., Wang, C., Wang, L., \& Liu, Y. (2013). Adaptive state of charge estimation for Li-ion batteries based on an unscented Kalman filter with an enhanced battery model. Energies, 6(8), 4134-4151.

[8] Hendricks, C., Williard, N., Mathew, S., \& Pecht, M. (2015). A failure modes, mechanisms, and effects analysis (FMMEA) of lithium-ion batteries. Journal of Power Sources, 297, 113-120.

[9] Liu, T., Ren, Y., Shen, Y., Zhao, S. X., Lin, Y., \& Nan, C. W. (2016). Achieving high capacity in bulk-type solid-state lithium ion battery based on Li6. 75La3Zr1. 75Ta0. 25012 electrolyte: Interfacial resistance. Journal of Power Sources, 324, 349357. 